OPEN ACCESS

Edited by:

David Rodriguez-Lazaro,

University of Burgos, Spain

Reviewed by:

Dario De Medici,

Istituto Superiore di Sanità, Italy

Young Min Kwon,

University of Arkansas, United States

*Correspondence:

Christopher J. Grim

christopher.grim@FDA.HHS.GOV

grim.christopher@gmail.com

Specialty section:

This article was submitted to

Food Microbiology,

a section of the journal

Frontiers in Microbiology

Received: 08 June 2017 Accepted: 04 August 2017

Published: 18 August 2017

Citation:

Grim CJ, Daquigan N, Lusk

Pfefer TS, Ottesen AR, White JR and Jarvis KG (2017) High-Resolution

Microbiome Profiling for Detection

and Tracking of Salmonella enterica.

Front. Microbiol. 8:1587.

doi: 10.3389/fmicb.2017.01587

\section{High-Resolution Microbiome Profiling for Detection and Tracking of Salmonella enterica}

\author{
Christopher J. Grim ${ }^{1 *}$, Ninalynn Daquigan ${ }^{1}$, Tina S. Lusk Pfefer ${ }^{2}$, Andrea R. Ottesen², \\ James R. White ${ }^{3}$ and Karen G. Jarvis ${ }^{1}$ \\ ${ }^{1}$ Center for Food Safety and Applied Nutrition, United States Food and Drug Administration, Laurel, MD, United States, \\ ${ }^{2}$ Center for Food Safety and Applied Nutrition, United States Food and Drug Administration, College Park, MD, \\ United States, ${ }^{3}$ Resphera Biosciences, Baltimore, MD, United States
}

16S rRNA community profiling continues to be a useful tool to study microbiome composition and dynamics, in part due to advances in next generation sequencing technology that translate into reductions in cost. Reliable taxonomic identification to the species-level, however, remains difficult, especially for short-read sequencing platforms, due to incomplete coverage of the 16S rRNA gene. This is especially true for Salmonella enterica, which is often found as a low abundant member of the microbial community, and is often found in combination with several other closely related enteric species. Here, we report on the evaluation and application of Resphera Insight, an ultra-high resolution taxonomic assignment algorithm for $16 \mathrm{~S}$ rRNA sequences to the species level. The analytical pipeline achieved $99.7 \%$ sensitivity to correctly identify S. enterica from WGS datasets extracted from the FDA GenomeTrakr Bioproject, while demonstrating 99.9\% specificity over other Enterobacteriaceae members. From low-diversity and low-complexity samples, namely ice cream, the algorithm achieved 100\% specificity and sensitivity for Salmonella detection. As demonstrated using cilantro and chili powder, for highly complex and diverse samples, especially those that contain closely related species, the detection threshold will likely have to be adjusted higher to account for misidentifications. We also demonstrate the utility of this approach to detect Salmonella in the clinical setting, in this case, bloodborne infections.

Keywords: 16S rRNA, community, profiling, microbiome, Salmonella, metagenomics

\section{INTRODUCTION}

Salmonella infection is a common bacterial disease throughout the developed and developing world, causing considerable morbidity and mortality. Salmonellosis can be categorized into two disease manifestations, a more common, and usually self-limiting, gastroenteritis due to non-typhoidal salmonellae (NTS) and the rarer, more severe, typhoid fever. Worldwide, it is estimated that NTS cause 94 million cases of gastroenteritis and 155,000 deaths per year (Majowicz et al., 2010), and in the United States, NTS are estimated to cause one million cases, 19,000 hospitalizations, and 380 deaths, annually (Dvir et al., 2014). The majority of non-typhoidal Salmonella cases are due to the consumption of contaminated food and water, and as such, this group of organisms is considered a major foodborne pathogen, occurring in almost all types of foods. Additionally, transmission of the organism can occur due to the handling of contaminated farm animals or reptiles. 
It is of paramount importance to rapidly and accurately identify foods that are contaminated with foodborne pathogens, such as Salmonella, as well as possible sources of contamination, to minimize public health burden. There are numerous culture-based detection and isolation protocols to detect foodborne pathogens, many of which are dependent on pathogen-commodity pairings, such as those in the Food and Drug Administration (FDA) Bacteriological Analytical Manual (BAM) and the United States Department of Agriculture (USDA) Microbiological Laboratory Guidebook (MLG). These methods rely on an array of selective and differential culture media to isolate colonies of the target pathogen, which are used for further characterization, such as sub-typing, serotyping, antibiotic susceptibility testing, and whole genome sequencing (WGS) (Humphries and Linscott, 2015). For example, detection of Salmonella generally involves an overnight non-selective enrichment, followed by an overnight selective enrichment in Rappaport Vassiliadis (RV) and Tetrathionate (TT) broths, followed by plating on selective media, such as Xylose lysine deoxycholate (XLD) or Xylose-Lysine-Tergitol 4 (XLT4) agar plates (Andrews et al., 2016). Suspect colonies are confirmed and then further characterized.

Although culture methods are the gold standard in microbiology due to their high sensitivity, current and emerging molecular methods, most notably, PCR, have greatly improved the time to detection for target microorganisms. Mass spectrometry-based methods, such as MALDI-TOF, have also shown great promise in detection and identification of bacterial pathogens. The use of WGS and the application of metagenomics have rapidly increased due to technological advances and cost-reduction in high-throughput next-generation sequencing (Kuczynski et al., 2011a). This technology has in turn become more accessible, and its application allows the characterization of an entire microbial community, in addition to multiple target pathogens (Jarvis et al., 2015; Ottesen et al., 2016).

Utilizing shotgun metagenomics and 16S rRNA gene sequencing is advantageous for microbiome profiling as well as targeted detection assays, because it is culture-independent and relatively unbiased compared to traditional culture methods that rely on highly selective media. While shotgun metagenomics can provide a better understanding of dominant microbial community members, such as identification to the species level, sub-typing and resistome characterization, this method is more expensive due to the requirement of deeper sequencing for higher genome coverage. Microbiome profiling through $16 \mathrm{~S}$ rRNA gene sequencing provides an economical approach to analyze microbial communities' dynamics, from a taxonomic perspective; tracking and identifying changes in that population structure in response to experimental variables. While this method is reliable for higher taxonomic levels, species-level inference with 16S rRNA gene sequencing has remained a significant challenge, particularly for accurate identification of Salmonella enterica and other enteric pathogens. Previously, we evaluated the performance of Resphera Insight, a high-resolution 16S rRNA sequence analysis pipeline, for identification of Listeria monocytogenes from naturally contaminated ice cream samples (Ottesen et al., 2016). We also recently applied this tool for tracking of $S$. enterica in a modified US FDA BAM protocol for detection of Salmonella through reduced enrichment (Daquigan et al., 2016). In this study, we performed an expanded evaluation of this algorithm for detection of S. enterica and evaluated factors affecting the identification of this pathogen in food and clinical samples.

\section{MATERIALS AND METHODS}

\section{Diagnostic True Positive Rate for S. enterica Detection}

To perform an evaluation of the species-level accuracy for S. enterica, 512 randomly selected S. enterica whole-genome shotgun sequencing datasets from the FDA GenomeTrakr Project (NCBI Project ID PRJNA183844) were obtained from the NCBI Sequence Read Archive (Supplementary Table S1). Paired-end MiSeq fastq sequences were first filtered based on quality (Q20) and length (150 bp), and then overlapping sequences were merged using FLASH (Magoc and Salzberg, 2011). Merged reads and any unmerged Read 1 sequences were then screened for $16 \mathrm{~S}$ rRNA fragments using Bowtie2 (Langmead and Salzberg, 2012) against a broad database of 16S rRNA sequences. An additional layer of BLAST-based filtering was used to confirm location-specific query matches to the S. enterica subsp. enterica serovar Typhimurium strain LT2 16S rRNA gene (NCBI accession NR_074910.1). Valid, filtered sequences were submitted to Resphera Insight (Baltimore, MD, United States ${ }^{1}$ ) for high-resolution taxonomic identification. Briefly, the tool relies on a manually curated 16S rRNA database of 11,000 species with a re-structured bacterial taxonomy and a hybrid global-local alignment strategy to assign sequences a high-resolution taxonomic lineage. The approach attempts to achieve species-level resolution when possible, but when the underlying statistical model indicates uncertainty in final species membership, the tool minimizes false positives by providing "ambiguous assignments" i.e., a list of species reflecting the uncertainty. For example, if a $16 \mathrm{~S}$ fragment is ambiguous between $S$. enterica and $S$. bongori, the algorithm will provide the assignment: "Salmonella_bongori:Salmonella_enterica."

Here, our statistic of performance sensitivity is the Diagnostic True Positive rate (DTP), defined as the percentage of 16S rRNA sequences that were correctly and unambiguously assigned to S. enterica. For comparison, we evaluated diagnostic performance for the RDP classifier and UCLUST-REF algorithms implemented within QIIME (Kuczynski et al., 2012; Navas-Molina et al., 2013), using default parameter settings. We also sought to apply the standard taxonomic characterization tools utilized by the MOTHUR (v1.35) and DADA2 (v1.0) packages (Schloss et al., 2009; Callahan et al., 2016); however, by default, these tools do not make species-level assignments. Therefore, they were excluded. The expanded validation of the Insight method was then performed on all available $S$. enterica isolates annotated in GenomeTrakr at the time of the study ( $n=12,090$, Supplementary Table S2). Of this expanded validation dataset,

${ }^{1}$ www.respherabio.com 
93 SRA datasets from GenomeTrakr were determined to be mis-annotated as Salmonella or reflected mixtures of microbial species based on WGS analysis using MetaPhlAn (Data not shown). To provide an unbiased evaluation of all three algorithms, four different size classes of sequencing read lengths were evaluated for the effect of this parameter on DTP. Further, DTP rates were determined for each position of the 16S rRNA gene, from nucleotide positions 25 to 1,000 . This approach was used to account for difference in 16S rRNA amplification and sequencing strategies.

\section{Evaluation of S. enterica False Positive Rate}

An evaluation of the false positive rate of Resphera Insight to accurately detect S. enterica was performed using 16S rRNA amplicon datasets reflecting raw milk cheese samples spiked with variable levels of Escherichia coli (O157:H7 or O103). In silico simulations for related Enterobacteriaceae were performed by first aligning primer sequences (V1-V3 or V3-V4 region) to corresponding reference $16 \mathrm{~S}$ rRNA genes to determine exact coordinates of the expected amplicon sequences, and subsequently generating sequences from those coordinates using a random nucleotide error rate of $0.5 \%$ (10,000 sequences per species for both regions).

\section{Bacterial Strains for Inoculation}

Cilantro was spiked with Salmonella Newport, S. Tennessee, or $S$. Thompson. Ice cream samples were spiked with $S$. Enteritidis or $S$. Typhimurium. Chili powder samples were spiked with $S$. Montevideo. Frozen stocks of S. enterica were streaked onto Trypticase Soy Agar plates (Difco ${ }^{\mathrm{TM}}$, Sparks, MD, United States) and incubated at $35^{\circ} \mathrm{C}$ overnight. Bacterial cell suspensions were prepared in $0.85 \%$ sterile saline to approximately 0.5 McFarland turbidity units, equivalent to $1 \times 10^{8} \mathrm{CFU} / \mathrm{mL}$, and serially diluted to approximately $28 \mathrm{CFU} / \mathrm{mL}$ for inoculation. Biosafety Level 2 practices, containment equipment, and facilities were employed for all experiments involving Biosafety Level 2 pathogens. All sample manipulations were performed within a certified Class II biological safety cabinet (BSC). Standard personnel protective equipment (PPE) was used, such as disposable gloves, laboratory coats, and eye protection.

\section{Preparation of Inoculated Food Matrices}

Cilantro was purchased from a local grocery store or provided by the Department of Agriculture and Rural Development in Lansing, Michigan, and then stored at $4^{\circ} \mathrm{C}$ until used. Ice cream, naturally contaminated with $L$. monocytogenes, was stored at $-20^{\circ} \mathrm{C}$ until used. Chili powder samples were supplied by the FDA Northeast Regional Laboratory. To prepare samples, $25 \mathrm{~g}$ of cilantro or chili powder, and $150 \mathrm{~g}$ of ice cream were aseptically portioned into sterile Whirlpak bags (Nasco; Fort Atkinson, WI, United States) and inoculated with Salmonella at a ratio of 1-2 CFU per gram for cilantro and ice cream and at four levels for chili powder, ultra-low (0.5 CFU/gm), low (5 CFU/gm), medium (51 CFU/gm), and high (512 CFU/gm). Cilantro samples were also "aged" at $4^{\circ} \mathrm{C}$ for $48-72 \mathrm{~h}$ to simulate natural contamination.

\section{Salmonella Enrichment from Food}

Following the FDA BAM method for Salmonella detection (Andrews et al., 2016), chili powder and aged cilantro samples were pre-enriched in a non-selective broth for $24 \mathrm{~h}$ (24-h samples), and then aliquots were transferred to selective RV and TT broths for a secondary $24 \mathrm{~h}$ enrichment (48-h samples). Ice cream samples were enriched for $48 \mathrm{~h}$ following the FDA BAM method for Listeria but without acriflavin, cycloheximide, and nalidixic acid (Hitchins et al., 2016). For all foods, a $10 \mu \mathrm{L}$ aliquot was streaked onto XLT4 (Becton, Dickinson and Company, Sparks, MD, United States) agar plates at two time points, 24 and $48 \mathrm{~h}$, and plates were incubated for $24 \mathrm{~h}$ at $35^{\circ} \mathrm{C}$. Suspect black colonies observed on XLT4 agar plates were confirmed as Salmonella using the VITEK 2 system (BioMérieux, France). Only samples with confirmed Salmonella were considered culture-positive. Microbiome cell pellets were also collected at each 0,24 , and 48 -h time point, centrifuged at $7,100 \times g$ for $30 \mathrm{~min}$, and stored at $-20^{\circ} \mathrm{C}$ until extracted from cell pellets. For chili powder, metagenomic samples were first vigorously mixed, passaged through Miracloth filter membrane (EMD Millipore, Billerica, MA, United States), and then sedimented at $200 \times g$ for $3 \mathrm{~min}$, to remove chili powder from bacterial cells, prior to harvesting the cell pellets by centrifugation, as described above.

\section{Sequencing of Microbiome Samples from Food}

Genomic DNA from cilantro microbiome samples was extracted and $16 \mathrm{~S}$ rRNA amplicon libraries were produced and sequenced as described in Daquigan et al. (2016). Genomic DNA from the ice cream microbiome samples was extracted and 16S rRNA amplicon libraries were produced and sequenced as described in Ottesen et al. (2016). Genomic DNA from chili powder samples was extracted using the DNeasy Blood and Tissue kit (Qiagen, Germantown, MD, United States). 16S rRNA community profile sequencing of chili powder microbiome samples was performed as described by Ottesen et al. (2016). Raw sequencing reads were subjected to preprocessing, as described in Section "Diagnostic True Positive Rate for S. enterica Detection," and submitted for high-resolution taxonomic identification.

\section{Analysis of Bacterial Blood Stream Infection 16S rRNA Dataset}

$16 S$ rRNA sequence data generated on the Illumina MiSeq platform utilizing paired-end sequencing were processed as follows: Raw overlapping paired-end reads were merged into consensus fragments by FLASH (Magoc and Salzberg, 2011) requiring a minimum 20 bp overlap with $5 \%$ maximum mismatch density, and subsequently filtered for quality (targeting error rates $<1 \%$ ) and length (minimum $200 \mathrm{bp}$ ) using Trimmomatic (Bolger et al., 2014) and QIIME (Caporaso et al., 2010a; Kuczynski et al., 2011b). Spurious hits to the PhiX control genome were identified using BLASTN and removed. Sequences were then trimmed of their associated primers, evaluated for chimeras with UCLUST (de novo mode) (Edgar et al., 2011), and screened for human-associated contaminant using Bowtie2 (Langmead and Salzberg, 2012) searches of NCBI Homo sapiens 
Annotation Release 106. Mitochondrial contaminants were detected and filtered using the RDP classifier (Wang et al., 2007) with a confidence threshold of $50 \%$, and passing high-quality $16 \mathrm{~S}$ sequences were assigned to a high-resolution taxonomic lineage using the Insight method. Species level assignments that were present with at least 10 reads in at least two negative control samples were designated as contaminants and removed from downstream analysis. Patients with fewer than 500 final sequences per duplicate MiSeq run were removed from analysis.

\section{RESULTS}

\section{Accuracy for S. enterica Identification}

We assessed how performance of three 16S rRNA signature identification algorithms, Insight, RDP, and UCLUST, changed according to changing sequencing read length and 16S rRNA gene position. Overall, across the $\mathrm{V} 1-\mathrm{V} 3$ region (16S rRNA gene positions 27-534), Resphera Insight achieved DTP rates up to 99.7\%, with improved accuracy correlated with increased read length (Figure 1A). For sequences less than $300 \mathrm{bp}$, sensitivity was significantly reduced. Among positions in the same region, RDP and UCLUST were unable to achieve a DTP above $11 \%$ for reads greater than $300 \mathrm{bp}$. Interestingly, the UCLUST method had improved sensitivity with shorter read lengths, though performance remained generally poor with a maximum DTP of $31.1 \%$ (Figure 1A). It should be noted that the DTP rates for all methods evaluated tended to dip in value across the $16 \mathrm{~S}$ rRNA sequence that corresponds to the $3^{\prime}$ end of the $\mathrm{V} 3$ region, and this reduction in diagnostic capability was more pronounced for shorter read lengths (Figure 1A).

As the Insight method had the highest DTP rates, an expanded evaluation was then performed on all $S$. enterica isolates annotated in GenomeTrakr available at the time ( $n=12,090$, Supplementary Table S2). In this larger evaluation, we observed a very consistent detection profile, nearly identical to that seen for the 512 random subset dataset (Figure 1A), including high accuracy within the first $250 \mathrm{bp}$ of the $16 \mathrm{~S}$ rRNA gene for sequences that are at least $300 \mathrm{bp}$ in length (Figure 1B).

\section{False Positive Rates for S. enterica Detection}

In addition to assessing the accuracy to detect Salmonella, we also sought to define the Type I error, or false positive rate, associated with detection of S. enterica; i.e., cases in which the algorithm incorrectly assigned a sequence to $S$. enterica. As $S$. enterica is closely related to $E$. coli, a false positive rate assessment was performed, utilizing 36 raw milk cheese samples spiked and selectively enriched with variable levels of $E$. coli, to establish how frequently an E. coli fragment would be incorrectly assigned to the target organism, Salmonella. Relative abundance estimates of Escherichia/Shigella ranged from 0.02 to $68.8 \%$ across the sample set, and we found a significant positive association between Escherichia/Shigella abundance and false positive assignments to S. enterica $\left(R^{2}=0.83, F\right.$-test $P<1 \mathrm{e}-4$; Figure 2$)$. Simply stated, the more Escherichia/Shigella 16S rRNA sequences contained in the microbiome sample, in terms of relative abundance, the more likely it was to produce a Salmonella false positive. However, using linear regression, the maximum predicted false positive rate was only $0.11 \%$, given a completely homogenous community comprised entirely of E. coli (Figure 2).

Moreover, in silico simulations in which sequencing reads are composed entirely of Enterobacteriaceae 16S rRNA gene sequences and no background flora, utilizing artificial reads from two amplicon regions, V1 - V3 and V3 - V4, of the 16S rRNA gene from pure isolates of 15 different Enterobacteriaceae members, indicate that false positive rates for Salmonella detection approach $0.2 \%$, when closely related members, such as Citrobacter and Enterobacter spp., are dominant members in a microbial community (Table 1). Interestingly, false positive rates were only observed for those Enterobacteriaceae species that are phylogenetically close to Salmonella, with, on average, slightly higher false positive rates observed for the V3 - V4 region (Table 1).

\section{Identification of S. enterica in Food Samples}

To demonstrate and evaluate the utility of Resphera Insight to detect $S$. enterica and characterize overall microbial community composition, we performed $16 \mathrm{~S}$ rRNA community profiling on microbial enrichment samples of (i) ice cream, (ii) cilantro, and (iii) chili powder. All three datasets included samples that were spiked with $S$. enterica and samples that were unspiked. Aliquots for $16 \mathrm{~S}$ rRNA community profiling and metagenomics were taken at procedural time points in the enrichment protocols, namely $0 \mathrm{~h}$ (unenriched, for all three samples), $24 \mathrm{~h}$ (following non-selective enrichment for cilantro and chili powder and midpoint of non-selective enrichment for ice cream), and $48 \mathrm{~h}$ (following non-selective enrichment for ice cream samples and following selective RV and TT enrichment for cilantro samples). Due to the low inoculation level used in the cilantro and ice cream experiments, Salmonella could not be detected in any of the 0 -h (un-enriched) samples, at the sequencing depth utilized (data not shown). Accordingly, 0 -h chili powder samples were also excluded from the analyses.

Salmonella enterica relative abundances in ice cream enrichments ranged as follows: 0.000 to $0.004 \%(24 \mathrm{~h}$, culture-negative); 0.000 to $0.008 \%$ (48 h, culture-negative); 7.018 to $26.742 \%$ ( $24 \mathrm{~h}$, culture-positive); and 13.272 to $30.842 \%$ (48 h, culture-positive) (Figure $3 \mathbf{A}$ and Supplementary Table S3). Ice cream samples showed low overall bacterial diversity and low initial bacterial load, approximately 200 CFUs per gram. The dominant organisms, during and following enrichment, were Lactococcus lactis, Bacillus spp., Enterococcus hermanniensis, and if spiked, S. enterica.

Among cilantro samples, S. enterica relative abundances ranged as follows: 0.000 to $0.140 \%$ ( $24 \mathrm{~h}$, culture-negative); 0.000 to $0.500 \%$ ( $48 \mathrm{~h}$, culture-negative); 0.024 to $58.568 \%$ (24 h, positive); and 2.757 to $96.936 \%$ (48 h, positive) (Figure 3B and Supplementary Table S3). The un-enriched microbiomes from cilantro samples had considerably higher bacterial loads, 

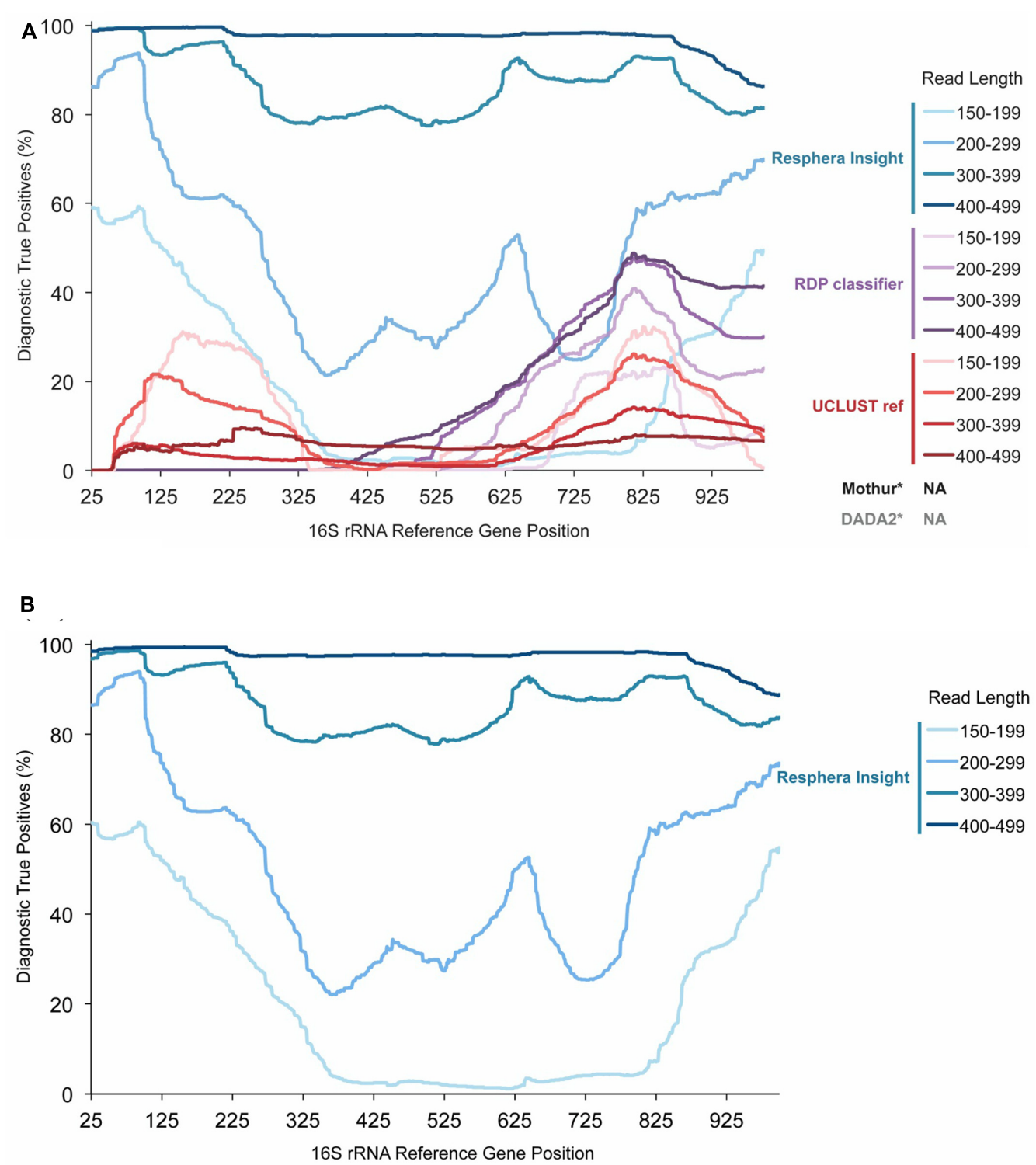

FIGURE 1 | Salmonella enterica Diagnostic True Positive Rate of Resphera Insight and 2 other taxonomy assignment tools. (A). Performance of the Insight method and other tools on 512 isolates of S. enterica. (B). Expanded validation of Resphera Insight on 12,090 isolates of S. enterica. Y-axis shows the Diagnostic True Positive Rate (DTP), i.e., the percentage of sequences assigned unambiguously to $S$. enterica. Lines display the average DTP for all reads covering each gene position for a given read length range. DTP rates are improved for Resphera Insight with longer sequences that cover the first 250 bp of the 165 rRNA gene. *The default versions of Mothur and DADA2 do not generate species level assignments.

$2 \times 10^{7} \mathrm{CFU}$ per gram on average, and higher diversity than ice cream (Figures 3A,B). In addition to Salmonella, the non-selective enrichment also enriched several other Enterobacteriaceae species, including Enterobacter cloacae, E. ludwigii, Morganella morganii, Pantoea spp., Erwinia spp. and Serratia spp. The relative abundance of several other taxa also increased from un-enriched to enriched time point samples, including Aeromonas, Lysinibacillus, and several Clostridium species, such as Clostridium perfringens and C. bifermentans. Pseudomonas was commonly found to be the dominant member of un-enriched cilantro samples (data not shown), and it persisted in the enriched samples, although its relative abundance decreased (Figure 3B).

For chili powder, S. enterica relative abundances ranged as follows: 0.010 to $0.226 \%$ in $24 \mathrm{~h}$ culture-negative samples and 7.392 to $79.920 \%$ in 24 h culture-positive samples (Figure 3C and Supplementary Table S3). The un-enriched microbiomes from chili powder samples were similar in terms of diversity to those of cilantro, but less abundant, $5 \times 10^{5} \mathrm{CFU}$ per gram on average. Similar to results from cilantro samples, the non-selective enrichment step not only enriched for S. enterica, but also enriched several other Enterobacteriaceae 


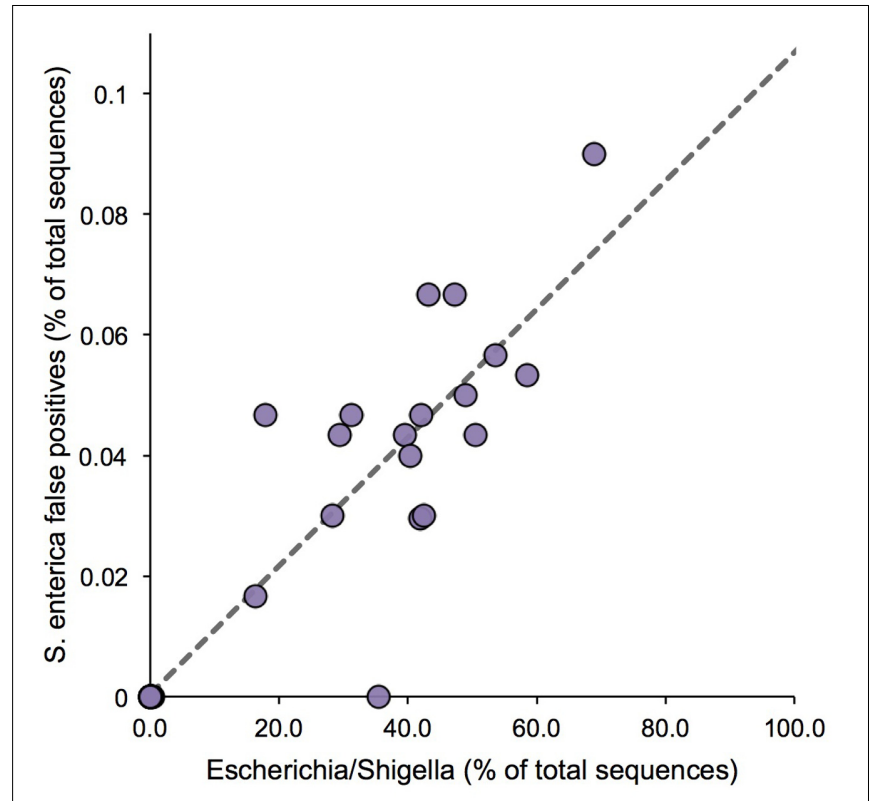

FIGURE 2 | False positive assignments to Salmonella for Resphera Insight. Sequencing of spiked samples of raw cheese milk demonstrates a positive correlation between Escherichia/Shigella abundance and false positives assignments to $S$. enterica. Linear regression (dashed line) estimates a maximum predicted $S$. enterica false positive rate of $0.11 \%$. species, in this case, Cronobacter, Enterobacter, Klebsiella, and Escherichia/Shigella. Many of these taxa were dominant members of the un-enriched microbiome, and they persisted through the non-selective enrichment step (Figure $3 \mathrm{C}$ ). The relative abundance of Enterococcus and Pediococcus also significantly increased from un-enriched to enriched time point samples (Figure 3C).

TABLE 1 | Salmonella enterica false positive rates for V1-V3 and V3-V4 $16 \mathrm{~S}$ rRNA amplicon regions.

\begin{tabular}{lcc}
\hline & \multicolumn{2}{c}{ S. enterica \% false positives } \\
\cline { 2 - 3 } Species & V1-V3 & V3-V4 \\
\hline Enterobacter cloacae & 0.030 & 0.200 \\
Leclercia adecarboxylata & 0.000 & 0.090 \\
Enterobacter aerogenes & 0.000 & 0.061 \\
Citrobacter amalonaticus & 0.040 & 0.040 \\
Enterobacter cancerogenus & 0.010 & 0.022 \\
Klebsiella pneumoniae & 0.000 & 0.021 \\
Enterobacter hormaechei & 0.040 & 0.020 \\
Escherichia coli & 0.020 & 0.010 \\
Citrobacter freundii & 0.000 & 0.010 \\
Proteus mirabilis & 0.000 & 0.000 \\
Serratia marcescens & 0.000 & 0.000 \\
Yersinia enterocolitica & 0.000 & 0.000 \\
Klebsiella oxytoca & 0.000 & 0.000 \\
Proteus mirabilis & 0.000 & 0.000 \\
Serratia marcescens & 0.000 & 0.000
\end{tabular}

Due to the intrinsic "noise" of 16S rRNA-based community profiling, investigators are forced to choose a minimum abundance threshold, defined as the percentage of reads assigned to $S$. enterica in a sample, to determine the outcome of a diagnostic assignment (presence/absence). Salmonella detection was evaluated using inoculated cilantro, chili powder and ice cream enrichments at four minimum abundance thresholds $(0.01,0.10,0.20$, and $0.25 \%)$ and compared to culture results. Sensitivity and specificity was $100 \%$ in all ice cream samples regardless of the Salmonella threshold utilized for analysis (Tables 2, 3). Using a $0.10 \%$ relative abundance threshold, the algorithm gave a $98 \%$ correct classification rate, or sensitivity, across all three commodities for Salmonella-positive $(n=174)$ samples (Table 2). A 96\% true negative rate was observed for Salmonella-negative $(n=120)$ samples using this threshold (Table 3), indicating the tool was both specific and sensitive for detecting Salmonella from food samples.

We observed low specificity to detect S. enterica in cilantro and chili powder samples when using the lower minimum abundance thresholds. For example, lowering this Salmonella threshold from 0.10 to $0.01 \%$ increased the sensitivity to $100 \%$ for all Salmonellapositive cilantro enrichments, but lowered the specificity to 38 and 33\% in Salmonella-negative 24 and $48 \mathrm{~h}$ enrichments, respectively (Table 3 ). Conversely, compared to the $0.10 \%$ threshold, a $0.20 \%$ threshold increased the specificity to $100 \%$, but decreased the sensitivity to $93 \%$ for 24 h cilantro enrichments. Similarly, increasing the threshold to $0.25 \%$ increased the specificity to $100 \%$ but also decreased the sensitivity to $91 \%$ (Table 3).

In Salmonella-negative 48-h enrichments from cilantro samples, the $89 \%$ specificity was consistent for the $0.10,0.20$, and $0.25 \%$ thresholds. One Salmonella-negative cilantro sample (48 h, RV enrichment) resulted in an unusually high S. enterica mis-assignment rate of $0.5 \%$; however, we did not observe a similar effect in its treatment pair ( $48 \mathrm{~h}$ TT broth). Further inspection of these pairs revealed that the false positive RV sample harbored a higher abundance of Enterobacter species (e.g., E. ludwigii, E. cloacae) compared to its TT pair (36.5 vs. $14.8 \%)$. This excess diversity of closely related species may have resulted in low frequency chimera formation during PCR not easily identified during preprocessing, which may be assigned to Salmonella.

A similar pattern was observed for the chili powder samples, even though the number of Salmonella-positive and -negative samples were low. This commodity also had a high number of other Enterobacteriaceae members, which may have resulted in chimeras not removed from the dataset during preprocessing, and contributed to a higher rate of misidentifications as S. enterica. This situation would also likely lead to a slight overrepresentation of the target organism, $S$. enterica in positive samples, as well.

\section{Application to Diagnosis of Bacterial Bloodstream Infection}

In addition to detection of $S$. enterica in food-associated microbial communities, we sought to apply this approach to 


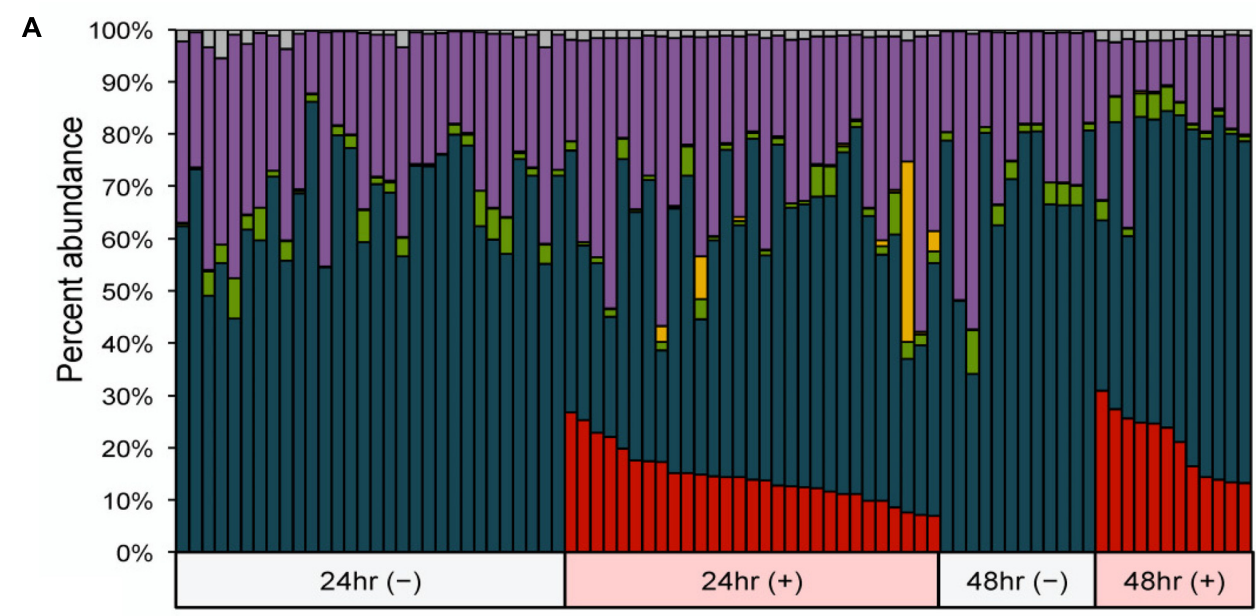

口Other taxa

口Bacillus spp.

口Leuconostoc mesenteroides

口Enterococcus hermanniensis

-Lactococcus lactis

-Salmonella enterica

B

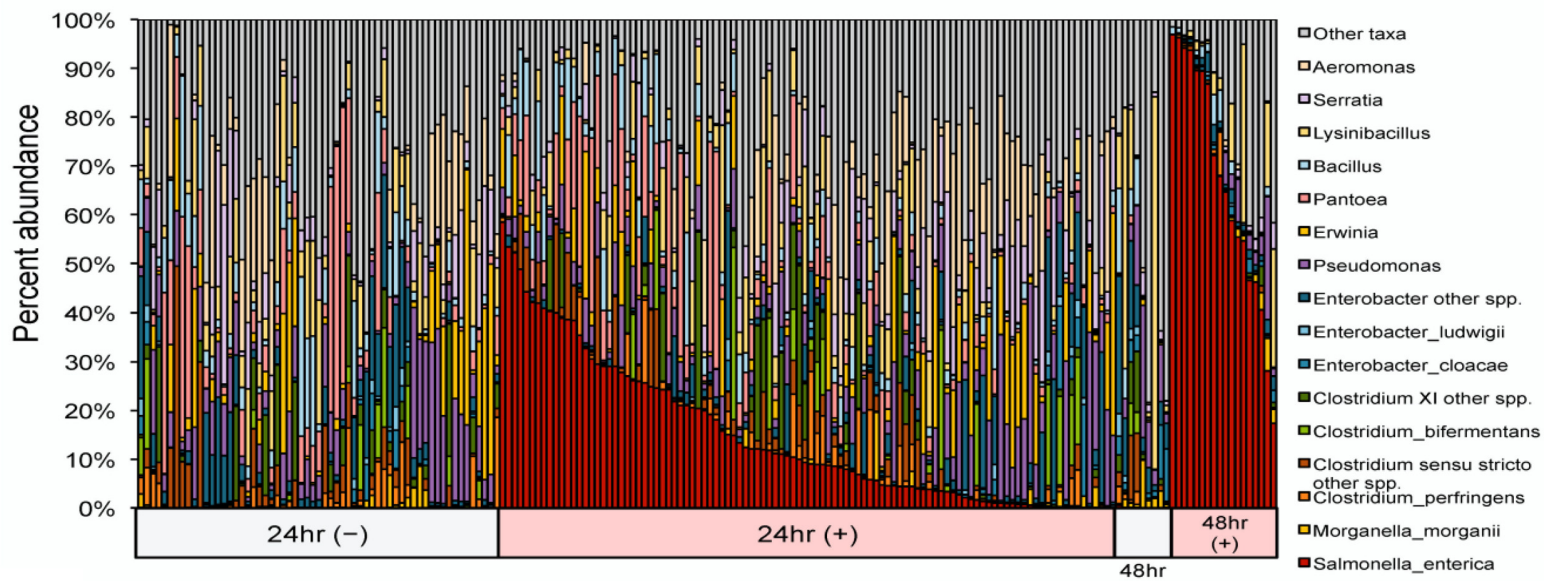

C

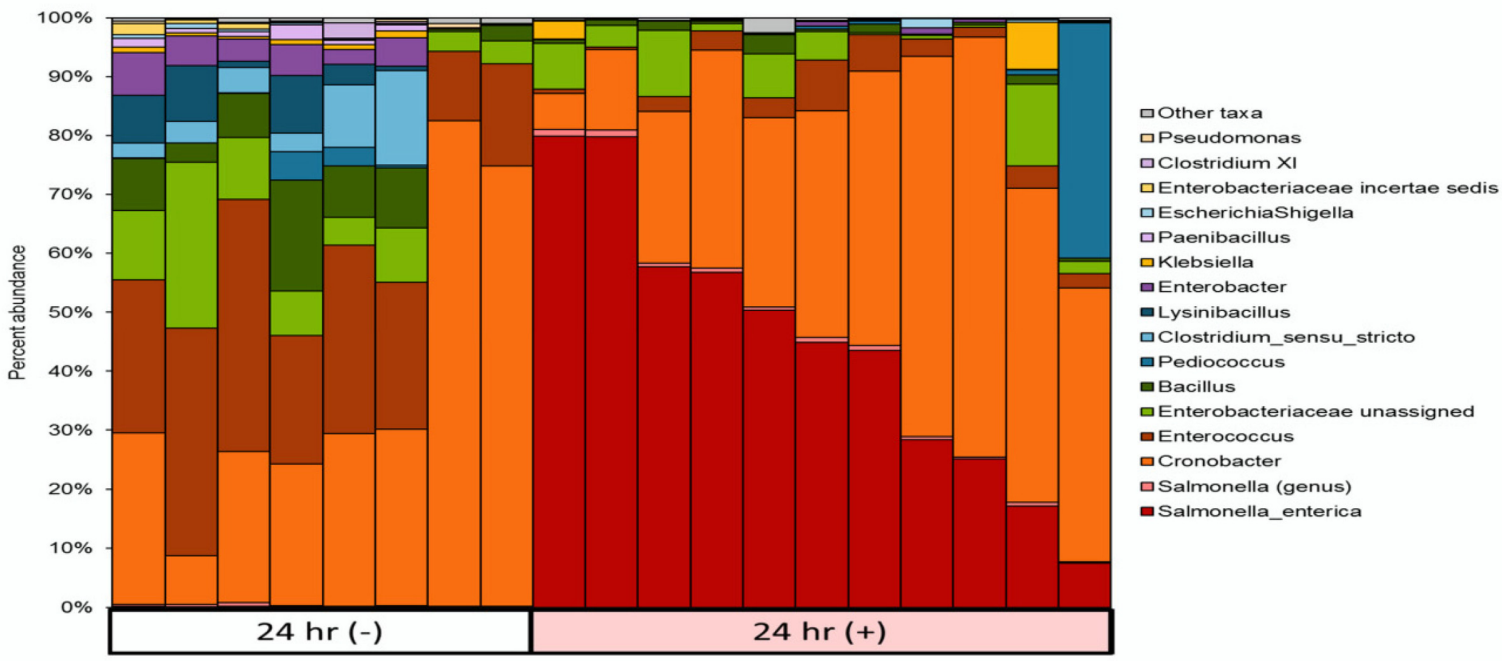

FIGURE 3 | Microbiome profiles for (A) ice cream and (B) cilantro samples after 24 and $48 \mathrm{~h}$ enrichments, and (C) chili powder samples after $24 \mathrm{~h}$ enrichments, sorted by Salmonella culture status $( \pm)$. We detect $S$. enterica with at least $0.1 \%$ abundance in $98 \%$ of Salmonella culture-positive samples. Ice cream samples are dominated by Lactococcus lactis, while cilantro- and chili powder-associated communities harbor increased overall diversity including members of Clostridia and Enterobacter. 
a recent study of children with severe febrile illness, reported by Decuypere et al. (2016). This study included confirmed culture positive cases of $S$. enterica bloodstream infections in a subset of children. We obtained and reprocessed the original 16S rRNA amplicon sequences from two replicate MiSeq runs (Run1 and Run2), requiring that passing samples have at least 500 high-quality $16 \mathrm{~S}$ rRNA sequences in both runs after removal of bacterial contaminants identified in negative controls (see Materials and Methods). This resulted in a final dataset of 26 pediatric patients, three of whom were blood culture positive for S. enterica (two for S. enterica serovar Enteritidis and one for S. enterica serovar Typhimurium).

All three patients positive for S. enterica were correctly identified in both MiSeq runs, all with $S$. enterica abundances greater than 6\% (Figure 4 and Supplementary Table S4). $S$. enterica sequences were also detected in three additional patients that were concordant across both replicate runs, however, each of these samples harbored less than $0.5 \%$ of the organism. Salmonella sequences were identified in the original study at a low level in the majority of samples, suggesting a contaminant background of Salmonella that would explain these culture negative findings.

\section{DISCUSSION}

Overall, these data provide a comprehensive assessment of the ability to detect $S$. enterica with high specificity and sensitivity using Resphera Insight high-resolution microbiome profiling.

TABLE 2 | Salmonella enterica sensitivity rates by limit of detection threshold $(0.01,0.10,0.20$, and $0.25 \%)$.

\begin{tabular}{|c|c|c|c|c|c|c|}
\hline \multirow[t]{2}{*}{$\begin{array}{l}\text { Sample } \\
\text { type }\end{array}$} & \multirow[t]{2}{*}{$\begin{array}{l}\text { Culture } \\
\text { result }\end{array}$} & \multirow[t]{2}{*}{$\begin{array}{l}\text { Enrichment } \\
\text { time }\end{array}$} & \multicolumn{4}{|c|}{$\begin{array}{c}\text { Sensitivity rate } \\
\text { by threshold }\end{array}$} \\
\hline & & & $0.01 \%$ & $0.10 \%$ & $0.20 \%$ & $0.25 \%$ \\
\hline \multirow[t]{2}{*}{ Cilantro } & Positive & $24 \mathrm{~h}(n=104)$ & $100 \%$ & $97 \%$ & $93 \%$ & $91 \%$ \\
\hline & & $48 \mathrm{~h}(n=18)$ & $100 \%$ & $100 \%$ & $100 \%$ & $100 \%$ \\
\hline \multirow[t]{2}{*}{ Ice cream } & Positive & $24 \mathrm{~h}(n=29)$ & $100 \%$ & $100 \%$ & $100 \%$ & $100 \%$ \\
\hline & & $48 \mathrm{~h}(n=12)$ & $100 \%$ & $100 \%$ & $100 \%$ & $100 \%$ \\
\hline \multirow[t]{2}{*}{ Chili powder } & Positive & $24 \mathrm{~h}(n=11)$ & $100 \%$ & $100 \%$ & $100 \%$ & $100 \%$ \\
\hline & & Total $(n=174)$ & $100 \%$ & $98 \%$ & $96 \%$ & $95 \%$ \\
\hline
\end{tabular}

TABLE 3 | Salmonella enterica specificity rates by limit of detection threshold (0.01, 0.10, 0.20, and 0.25\%).

\begin{tabular}{|c|c|c|c|c|c|c|}
\hline & & & \multicolumn{4}{|c|}{ Specificity rate by threshold } \\
\hline & & & $0.01 \%$ & $0.10 \%$ & $0.20 \%$ & $0.25 \%$ \\
\hline \multirow[t]{2}{*}{ Cilantro } & Negative & $24 \mathrm{~h}(n=61)$ & $38 \%$ & $98 \%$ & $100 \%$ & $100 \%$ \\
\hline & & $48 \mathrm{~h}(n=9)$ & $33 \%$ & $89 \%$ & $89 \%$ & $89 \%$ \\
\hline \multirow[t]{2}{*}{ Ice cream } & Negative & $24 \mathrm{~h}(n=30)$ & $100 \%$ & $100 \%$ & $100 \%$ & $100 \%$ \\
\hline & & $48 \mathrm{~h}(n=12)$ & $100 \%$ & $100 \%$ & $100 \%$ & $100 \%$ \\
\hline \multirow[t]{2}{*}{ Chili powder } & Negative & $24 \mathrm{~h}(n=8)$ & $12.5 \%$ & $62.5 \%$ & $87.5 \%$ & $100 \%$ \\
\hline & & Total $(n=120)$ & $58 \%$ & $96 \%$ & $98 \%$ & $99 \%$ \\
\hline
\end{tabular}

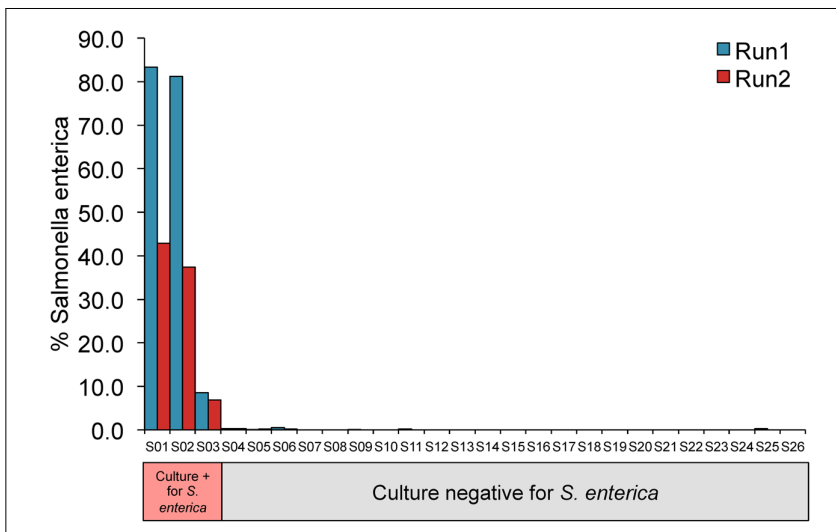

FIGURE 4 | 16S rRNA profiling of blood samples are consistent with Salmonella culture results. $Y$-axis displays percentage of total sequences assigned to $S$. enterica.

When compared against two commonly used algorithms for assigning taxonomy within QIIME (Caporaso et al., 2010b), the tool performed significantly better in terms of Diagnostic True Positive rate, which translates into a higher proportion of true $S$. enterica sequences being assigned correctly to this species. Unclassified assignments with existing tools continue to be an area of concern even though the amount of $16 \mathrm{~S}$ rRNA sequences grows exponentially in this WGS era, highlighting the need for tools such as this high-resolution microbiome profiling algorithm and database to help accurately classify, or assign taxonomy, to large microbiome datasets.

We observed a similarly high specificity for S. enterica, or true negative rate. Using in silico simulations of other Enterobacteriaceae and raw milk cheese spiked with E. coli, we demonstrated that the diagnostic false positive rate for $S$. enterica was never above $0.11 \%$. This high specificity was confirmed with low-diversity ice cream samples that were culture-negative for Salmonella. However, when microbiome samples, such as cilantro and chili powder, contained a more complex microbiome, including several closely related enteric species, the specificity of the tool fell dramatically at the lowest minimum abundance detection threshold tested. In terms of bacterial biomass, it is notable that the 24-h Salmonella positive cilantro samples had levels of Salmonella ranging from 7.44 to $8.74 \log$ MPN -g cilantro with corresponding background bacterial concentrations of 9 to $10 \log$ CFU -g (Daquigan et al., 2016). In comparison, the 24-h ice cream samples with less diverse microbiomes, harbored bacterial concentrations of approximately $6.43 \log$ MPN $-g$ and 10.54 $\log$ CFU - $g$ for Salmonella and background microorganisms, respectively. This further demonstrates the higher impact of microbiome diversity on specificity even when concentrations of cultured background microorganisms and Salmonella were similar. We suspect that the presence of closely related species impacts the specificity of the algorithm through more false positives to Salmonella due to "noisy" low frequency sequences that are mis-identified and a higher formation of chimeras during PCR that may not be adequately filtered by preprocessing. However, it should be mentioned that these challenges are a 
characteristic of the dataset and would apply to all analytical tools used.

The decrease in specificity could be resolved, by moving the minimum abundance threshold higher. However, this resulted in a loss of sensitivity with samples in which the relative abundance was below the minimum abundance threshold. Fortunately, 16S rRNA metagenomics gives a complete picture of the microbial community sequenced, so that the detection threshold can be adjusted accordingly based on the microbial composition. Based on this study, a $0.20 \%$ relative abundance threshold for S. enterica was established with an overall sensitivity of $96 \%$ and specificity of $98 \%$. When this $0.20 \%$ threshold was used to detect Salmonella from bloodstream samples, all three culture-positive patients were identified, supporting the notion that accurate identification of Salmonella can be performed in clinically relevant scenarios, as well as food safety surveillance.

Given the increasing level of interest in microbiome analysis by food safety stakeholders, this study suggests that microbiome sequencing diagnostic tools to identify pathogens need to be carefully designed and implemented, with rigorous evaluations to determine sensitivity and specificity, as demonstrated here. In addition to downstream analytical tool choice, all other aspects of this workflow, such as sample collection and DNA extraction, 16S rRNA amplification and sequencing strategy, must also be evaluated. For example, as demonstrated in this study with Salmonella and, in a previous study of Listeria, targeting the $\mathrm{V} 1$ to $\mathrm{V} 3$ region of the $16 \mathrm{~S}$ rRNA gene improves sensitivity and specificity of detection of these pathogens (Ottesen et al., 2016). However, this may not be the case with all organisms; for example, Bacillus anthracis can be distinguished from members of the $B$. cereus group by targeting the V6 region (Chakravorty et al., 2007).

Due to intrinsic noise in $16 \mathrm{~S}$ rRNA community profiling, even tools such as Resphera Insight, which demonstrated high

\section{REFERENCES}

Andrews, W. H., Wang, H., Jacobson, A., and Hammack, T. (2016). "BAM: Salmonella," in Bacteriological Analytical Manual, ed. Food and Drug Administration (Silver Spring, MD: USFDA).

Bolger, A. M., Lohse, M., and Usadel, B. (2014). Trimmomatic: a flexible trimmer for Illumina sequence data. Bioinformatics 30, 2114-2120. doi: 10.1093/bioinformatics/btu170

Callahan, B. J., Mcmurdie, P. J., Rosen, M. J., Han, A. W., Johnson, A. J., and Holmes, S. P. (2016). DADA2: high-resolution sample inference from Illumina amplicon data. Nat. Methods 13, 581-583. doi: 10.1038/nmeth.3869

Caporaso, J. G., Bittinger, K., Bushman, F. D., Desantis, T. Z., Andersen, G. L., and Knight, R. (2010a). PyNAST: a flexible tool for aligning sequences to a template alignment. Bioinformatics 26, 266-267. doi: 10.1093/bioinformatics/ btp636

Caporaso, J. G., Kuczynski, J., Stombaugh, J., Bittinger, K., Bushman, F. D., Costello, E. K., et al. (2010b). QIIME allows analysis of high-throughput community sequencing data. Nat. Methods 7, 335-336. doi: 10.1038/nmeth. f.303

Chakravorty, S., Helb, D., Burday, M., Connell, N., and Alland, D. (2007). A detailed analysis of $16 \mathrm{~S}$ ribosomal RNA gene segments for the diagnosis of pathogenic bacteria. J. Microbiol. Methods 69, 330-339. doi: 10.1016/j.mimet. 2007.02.005 specificity and sensitivity, show limitations and challenges in their usage. As shown in this study, detection of S. enterica will depend not only on its absolute abundance, but also its relative abundance compared to the background microbial population and the complexity of that population. False positive assignments, though infrequent, do occur suggesting that identification of S. enterica below $0.20 \%$ abundance may be challenging for some samples in which other closely related Enterobacteriaceae members dominate a community. However, for microbiological research, the ability to characterize the microbial population and its changes can be beneficial for studying how experimental parameters and sample sources, such as different food matrices, affect target recovery.

\section{AUTHOR CONTRIBUTIONS}

CG, ND, and JW drafted and wrote the manuscript. CG, ND, TL, $\mathrm{AO}$, and $\mathrm{KJ}$ designed and conducted the experiments. CG, ND, KJ, and JW analyzed and interpreted the data. JW performed the bioinformatics analyses. All authors read and approved the final manuscript.

\section{FUNDING}

This work was funded by the United States Food and Drug Administration.

\section{SUPPLEMENTARY MATERIAL}

The Supplementary Material for this article can be found online at: http://journal.frontiersin.org/article/10.3389/fmicb. 2017.01587/full\#supplementary-material

Daquigan, N., Grim, C. J., White, J. R., Hanes, D. E., and Jarvis, K. G. (2016). Early recovery of Salmonella from food using a 6-hour non-selective pre-enrichment and reformulation of tetrathionate broth. Front. Microbiol. 7:2103. doi: 10.3389/ fmicb.2016.02103

Decuypere, S., Meehan, C. J., Van Puyvelde, S., De Block, T., Maltha, J., Palpouguini, L., et al. (2016). Diagnosis of bacterial bloodstream infections: a 16S metagenomics approach. PLoS Negl. Trop. Dis. 10:e0004470. doi: 10.1371/ journal.pntd.0004470

Dvir, M., Peretz, A., Haitin, Y., and Attali, B. (2014). Recent molecular insights from mutated IKS channels in cardiac arrhythmia. Curr. Opin. Pharmacol. 15, 74-82. doi: 10.1016/j.coph.2013.12.004

Edgar, R. C., Haas, B. J., Clemente, J. C., Quince, C., and Knight, R. (2011). UCHIME improves sensitivity and speed of chimera detection. Bioinformatics 27, 2194-2200. doi: 10.1093/bioinformatics/btr381

Hitchins, A. D., Jinneman, K. C., and Chen, Y. (2016). "BAM: detection and enumeration of Listeria monocytogenes," in Bacteriological Analytical Manual, ed. Food and Drug Administration (Silver Spring, MD: USFDA).

Humphries, R. M., and Linscott, A. J. (2015). Laboratory diagnosis of bacterial gastroenteritis. Clin. Microbiol. Rev. 28, 3-31. doi: 10.1128/CMR.00073-14

Jarvis, K. G., White, J. R., Grim, C. J., Ewing, L., Ottesen, A. R., Beaubrun, J. J., et al. (2015). Cilantro microbiome before and after nonselective pre-enrichment for Salmonella using 16S rRNA and metagenomic sequencing. BMC Microbiol. 15:160. doi: 10.1186/s12866-015-0497-2 
Kuczynski, J., Lauber, C. L., Walters, W. A., Parfrey, L. W., Clemente, J. C., Gevers, D., et al. (2011a). Experimental and analytical tools for studying the human microbiome. Nat. Rev. Genet. 13, 47-58. doi: 10.1038/ nrg3129

Kuczynski, J., Stombaugh, J., Walters, W. A., Gonzalez, A., Caporaso, J. G., and Knight, R. (2011b). Using QIIME to analyze 16S rRNA gene sequences from microbial communities. Curr. Protoc. Bioinform. 36, 10.7.1-10.7.20. doi: 10.1002/0471250953.bi1007s36

Kuczynski, J., Stombaugh, J., Walters, W. A., Gonzalez, A., Caporaso, J. G., and Knight, R. (2012). Using QIIME to analyze 16S rRNA gene sequences from microbial communities. Curr. Protoc. Microbiol. 36, 10.7.1-10.7.20. doi: 10.1002/9780471729259.mc01e05s27

Langmead, B., and Salzberg, S. L. (2012). Fast gapped-read alignment with Bowtie 2. Nat. Methods 9, 357-359. doi: 10.1038/nmeth.1923

Magoc, T., and Salzberg, S. L. (2011). FLASH: fast length adjustment of short reads to improve genome assemblies. Bioinformatics 27, 2957-2963. doi: 10.1093/ bioinformatics/btr507

Majowicz, S. E., Musto, J., Scallan, E., Angulo, F. J., Kirk, M., O’brien, S. J., et al. (2010). The global burden of nontyphoidal Salmonella gastroenteritis. Clin. Infect. Dis. 50, 882-889. doi: 10.1086/650733

Navas-Molina, J. A., Peralta-Sanchez, J. M., Gonzalez, A., Mcmurdie, P. J., VazquezBaeza, Y., Xu, Z., et al. (2013). Advancing our understanding of the human microbiome using QIIME. Methods Enzymol. 531, 371-444. doi: 10.1016/B9780-12-407863-5.00019-8

Ottesen, A., Ramachandran, P., Reed, E., White, J. R., Hasan, N., Subramanian, P., et al. (2016). Enrichment dynamics of Listeria monocytogenes and the associated microbiome from naturally contaminated ice cream linked to a listeriosis outbreak. BMC Microbiol. 16:275. doi: 10.1186/s12866-016-0894-1
Schloss, P. D., Westcott, S. L., Ryabin, T., Hall, J. R., Hartmann, M., Hollister, E. B., et al. (2009). Introducing mothur: open-source, platform-independent, community-supported software for describing and comparing microbial communities. Appl. Environ. Microbiol. 75, 7537-7541. doi: 10.1128/AEM. 01541-09

Wang, Q., Garrity, G. M., Tiedje, J. M., and Cole, J. R. (2007). Naive Bayesian classifier for rapid assignment of rRNA sequences into the new bacterial taxonomy. Appl. Environ. Microbiol. 73, 5261-5267. doi: 10.1128/AEM.00062-07

Disclaimer: Use of trade names and commercial sources is for identification purposes only and does not imply endorsement by FDA or the United States Department of Health and Human Services.

Conflict of Interest Statement: JW is founder of Resphera Biosciences and has an equity position in the company.

The other authors declare that the research was conducted in the absence of any commercial or financial relationships that could be construed as a potential conflict of interest.

Copyright (c) 2017 Grim, Daquigan, Lusk Pfefer, Ottesen, White and Jarvis. This is an open-access article distributed under the terms of the Creative Commons Attribution License (CC BY). The use, distribution or reproduction in other forums is permitted, provided the original author(s) or licensor are credited and that the original publication in this journal is cited, in accordance with accepted academic practice. No use, distribution or reproduction is permitted which does not comply with these terms. 